\title{
Correction to: The Kendrick modelling platform: language abstractions and tools for epidemiology
}

Mai Anh BUI T $1^{*}$, Nick Papoulias ${ }^{2}$, Serge Stinckwich ${ }^{3,4,5}$, Mikal Ziane ${ }^{6,7}$ and Benjamin Roche ${ }^{3}$

\section{Correction to: BMC Bioinf (2019) 20:312 \\ https://doi.org/10.1186/s12859-019-2843-0}

Following publication of the original article [1], the author noticed that the following lines were missing from the published article. The original article has been corrected.

In the 'Results' section under heading 'Case study I: Measles', the following lines are missing in the published article:

1. line 'KendrickModel SEIR' should be inserted before the following:

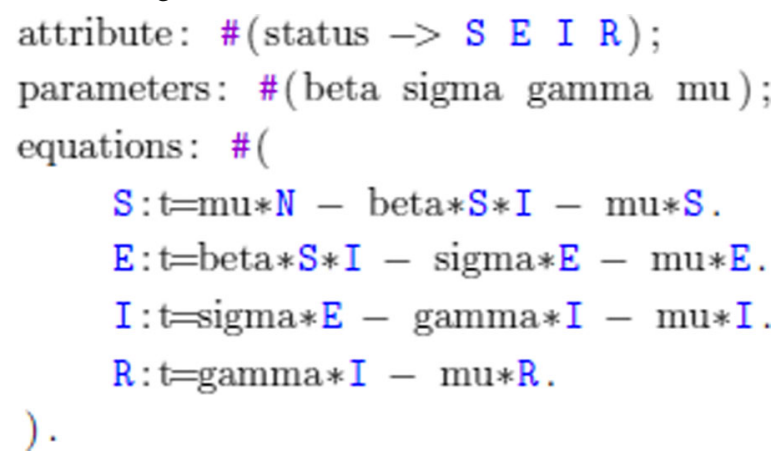

2. line 'Simulation MeaslesRKSim rungeKutta' should be inserted before the following:

scenarios: \#(MeaslesParameters MeaslesPopulation); from: 0.0 ;

to: 150 ; step: 1 .

Visualization MeaslesDiagramViz diagram for : 'MeaslesRKSim ';

xLabel: 'Time (days)';

exportToPng.

3. line 'KendrickModel SIR' should be inserted before the following:

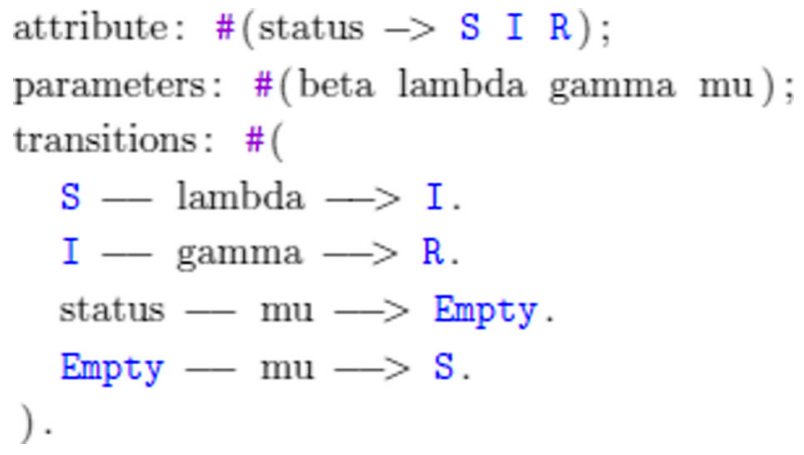

4. line 'Experiment PopRateAnalysis' should be inserted before the following:

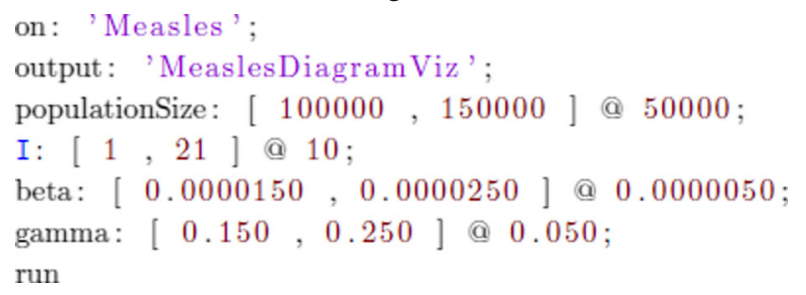

* Correspondence: anhbtm@soict.hust.edu.vn

${ }^{1}$ Software Engineering Department, School of Information and

Communication Technology, Hanoi University of Science and Technology,

Hanoi, Vietnam

Full list of author information is available at the end of the article

๑ The Author(s). 2019 Open Access This article is distributed under the terms of the Creative Commons Attribution 4.0 International License (http://creativecommons.org/licenses/by/4.0/), which permits unrestricted use, distribution, and reproduction in any medium, provided you give appropriate credit to the original author(s) and the source, provide a link to the Creative Commons license, and indicate if changes were made. The Creative Commons Public Domain Dedication waiver (http://creativecommons.org/publicdomain/zero/1.0/) applies to the data made available in this article, unless otherwise stated. 


\section{Author details}

${ }^{1}$ Software Engineering Department, School of Information and

Communication Technology, Hanoi University of Science and Technology, Hanoi, Vietnam. ${ }^{2}$ Université de La Rochelle, UMR 7266 LIENSs, CNRS, La

Rochelle, France. ${ }^{3}$ Sorbonne Université, IRD, Unité de Modélisation

Mathématiques et Informatique des Systèmes Complexes, UMMISCO,

F-93143 Bondy, France. ${ }^{4}$ Université de Yaoundé I, IRD, UMMISCO, Yaoundé, Cameroon. ${ }^{5}$ Université de Caen Normandie, Caen, France. ${ }^{6}$ Université de Paris, Paris, France. ${ }^{7}$ Sorbonne Université, CNRS, Laboratoire d'Informatique de Paris 6, LIP6, F-75005 Paris, France.

Received: 30 June 2019 Accepted: 4 July 2019

Published online: 27 August 2019

\section{Reference}

1. BUI T MA, et al. The Kendrick modelling platform: language abstractions and tools for epidemiology. BMC Bioinf. 2019;20:312. https://doi.org/10.1186/s12 859-019-2843-0. 